\title{
Negative linear, in-plane zero and phase-transition-induced negative volume expansion in cranswickite-type $\mathrm{MgSO}_{4} \cdot 4 \mathrm{D}_{2} \mathrm{O}$
}

\author{
Johannes M. Meusburger ${ }^{1,2,3}$, Karen A. Hudson-Edwards ${ }^{1}$, Chiu C. Tang ${ }^{2}$, Eamonn Connolly ${ }^{2}$, Rich A. Crane ${ }^{1}$, \\ A. Dominic Fortes ${ }^{3}$ \\ ${ }^{1}$ Camborne School of Mines and Environment and Sustainability Institute, Tremough Campus, \\ University of Exeter, Penryn TR10 9EZ, UK \\ ${ }^{2}$ Diamond Light Source, Harwell Science and Innovation Campus, Fermi Avenue, Didcot OX11 ODE, UK \\ ${ }^{3}$ ISIS Neutron and Muon Source, STFC Rutherford Appleton Laboratory, Harwell Science and Innovation Campus, Chilton, Didcot, \\ Oxfordshire, OX11 0QX, UK \\ johannes.meusburger@stfc.ac.uk
}

Hydrated sulfates are likely to be the dominant water reservoir in the equatorial region of Mars [1] forming massive, stratified deposits [2]. Hence their detailed mineralogical characterisation is critical in order to understand the aqueous history as well as the present-day equatorial water cycle of our neighbouring planet. Due to significant spectral similarities between sulfates of different chemical composition and degrees of hydration, attempts to constrain the exact nature of the polyhydrated sulfates from remote sensing data has proven to be challenging [3]. In-situ analysis by means of Raman spectroscopy and X-ray diffraction in Rover missions, however, appears to be a promising approach to provide insight on the mineralogy of these deposits. In order to facilitate such a phase analysis, there is a major interest in the thermal expansion and vibrational characteristics, and structural stability of candidate minerals at temperatures relevant to the martian surface.

To this end, we carried out a systematic study on various magnesium sulfate hydrates and related compounds and, notably for the first time, managed to synthesise cranswickite-type $\mathrm{MgSO}_{4} \cdot 4 \mathrm{D}_{2} \mathrm{O}$ in the laboratory. Subsequently, we determined its thermal expansion at temperatures ranging from 340 to $20 \mathrm{~K}$ by means of time-of-flight neutron powder diffraction at the HRPD instrument (ISIS facility, UK) and from 300 to $80 \mathrm{~K}$ using high-resolution synchrotron X-ray powder diffraction at the I11 beamline (Diamond Light Source, UK).

The cranswickite samples studied revealed negative linear thermal expansion // $c$ of approximately the same magnitude as the the positive linear thermal expansion // $a$, resulting in a net-zero thermal expansion in the $a c$ plane over the entire temperature range under investigation. At $340 \mathrm{~K}$ cranswickite (space group $C 2 / c$ ) underwent a polymorphic phase transition to starkeyite (space group $P 2_{1} / n$ ). The phase transition proceeded sluggishly and took several hours to complete. Most importantly, the cranswickite-to-starkeyite transition is accompanied by a volume reduction $(\Delta V \approx-4.5 \%)$, thus contradicting the general expectation that a less dense polymorph is formed at higher temperatures. To the best of our knowledge such interesting behaviour has so far just been observed for $\mathrm{ThSiO}_{4}$ and isotypic $\mathrm{PaSiO}_{4}$, albeit at substantially higher temperatures of approximately $1520 \mathrm{~K}$ [4].

[1] Feldman, W., Mellon, M., Maurice, S., Prettyman, T., Carey, B., Vaniman, D., Bish, D., Claire, F., Chipera, S., Kargel, J., Elphic, R., Funsten, H. (2004). Geophys. Res. Lett. 31, 10.1029/2004GL020181.

[2] Roach, L., Mustard, J., Swayze, G., Milliken, R., Bishop, J., Murchie, S., Lichtenberg, K. (2010). Icarus 206, $253-268$. 10.1016/j.icarus.2009.09.003.

[3] Bishop, J. L., et al. (2009). J. Geophys. Res. 114, E00D09, 10.1029/2009JE003352.

[4] Keller, C., (1963). Nukleonik 5, 41-48

Keywords: negative thermal expansion, sulfate, Mars, phase-transition

JMM acknowledges funding from an ISIS Facility Development and Utilisation Studentship (50\%) and the University of Exeter (50 $\%)$. 